"This is the peer reviewed version of the following article: Hong, K. J., Peng, B. and Zhang, X. Economic Record (2015) which has been published in final form at http://onlinelibrary.wiley.com/doi/10.1111/1475-4932.12208/abstract. This article may be used for non-commercial purposes in accordance with Wiley Terms and Conditions for SelfArchiving." 


\title{
Capturing the Impact of Unobserved Industry-Wide Shocks on Stock Returns with Panel Data Model*
}

\author{
KiHoon Jimmy Hong ${ }^{\mathrm{a}}$, Bin Peng ${ }^{\mathrm{b}}$ and Xiaohui Zhang ${ }^{\mathrm{c}, ~}{ }^{\dagger}$ \\ ${ }^{a}$ Hongik University, College of Business, Seoul, 121-791, Republic of Korea \\ ${ }^{\mathrm{b}}$ Economics Discipline Group, University of Technology, Sydney, Australia \\ c School of Management and Governance, Murdoch University, Perth, Australia
}

\begin{abstract}
Unobserved industry-wide common shocks cause issue of cross-sectional dependence (CSD) in panel data modelling of stock returns. In this study we apply two econometric techniques: SUR approach and a Bayesian estimator for panel data model with factor structural errors, to allow for CSD within a particular industry. By applying these models to monthly stock returns of S\&P100 companies from six industries over 10 years, we can capture and measure the heterogeneous impacts of not only observed individual company accounting fundamentals and market-wide common shocks, but also the unobservable industry-wide common shocks. Results from the empirical study show that the impacts from both observed factors and unobserved industry-wide common shocks vary markedly across companies. After controlling observed accounting fundamentals and market-wide common factors, considerable proportions of variations in stock returns can be attributed to unobservable industry-wide common shocks.
\end{abstract}

JEL classification: C11, C13, G12

Keywords: Cross-Sectional Dependence; Factor Structural Error; Common Shocks; Stock Returns; Accounting Fundamentals; Bayesian Gibbs Sampler

\footnotetext{
* We would like to thank Steve Satchell, Yong Woong Lee and seminar participants at University of Technology, Sydney for their useful comments and discussions. We gratefully acknowledge financial support from University of Technology, Sydney, Business School. All errors remain our own.

† Corresponding author. Tel: +61 8 93606217, Email: xiaohui.zhang@murdoch.edu.au
} 


\section{Introduction}

There are a considerable amount of factors that can influence stock price movements. Both practitioners and academics have devoted much time to explaining and predicting stock price movements. Fundamental analysts primarily use accounting information to study a company's underlying indicators of profit, such as earnings, dividends, new products and research and development (R\&D). They investigate the financial statements of a company and its competitors to estimate the company's future value. The advantage of fundamental analysis is that it has an intuitive explanatory link to stock price movements. It ought to characterize the long-term, fundamental value of a stock, at least in theory. If the information on financial statements accurately reveals the fundamental value of a company, the accounting information should explain a significant proportion of stock price movements.

From another aspect, the Capital Asset Pricing Model (CAPM) theory states that the market excess return captures the systematic exposure of individual stock's excess return. Therefore, market index return is likely to have statistical significance in explaining stock price movements (see Lessard, 1974). If market-wide common shocks exist and are represented by market index return, the same could happen within an industry. In fact, results from previous studies show that the capability of accounting variables, together with marketwide impact, such as S\&P500, is limited in explaining stock price movements (c.f. Chen and Zhang, 2007; Bettmanet, et al., 2009). This, to some extent, demonstrates the existence of other factors, such as industry-wide common shocks, that influence stock price movements.

By applying an extended Cournot and Bertrand competition model, Hao et al. (2011) theoretically and empirically found that stock price movements are sensitive to industry-level news, and that the returns of less profitable companies in an industry are more sensitive to industry-level news than those of the more profitable companies. In the empirical analysis, they used equally weighted returns of all companies in a particular industry as a proxy for industry-level news. Harford (2005) also noted the existence of industry-specific economic shocks, and found that the proxy variables for industry-specific economic shocks are highly correlated within an industry, which may cause multicollinearity, if simultaneously included in a regression model. To address this, Harford (2005) extracted the first principal component from seven observable economic shock variables.

In practice most, if not all, common shock variables are unobservable or have no reasonable proxy, so the proxy variable method or principal component analysis may not work. These unobserved industry-wide common shocks cause the issue of cross-sectional 
dependence (CSD) in panel data modelling of stock price movements. However, to the authors' best knowledge, very few studies in the literature have ever considered it. To fill in the gap of literature, in this study we apply different econometric techniques to allow for the CSD when modelling stock price movements within a particular industry.

One of the classic models to allow for CSD in econometrics is the Seemingly Unrelated Regression (SUR) approach, first introduced by Zellner (1962). In SUR model all regressors are assumed exogenous and the asymptotic properties of estimates are derived for fixed $N$ (the number of cross-sectional individuals) and large $T$ (the number of time periods). Therefore, neglecting CSD has no impact on the first-order properties of standard panel estimators.

In the last decades, considerable research has been devoted to characterizing CSD in panel data using the factor structure approach, which assumes that the disturbance term contains a finite number of unobserved factors that influence each individual but with different intensities, that is, all individuals are influenced by some unobserved common shocks but they have heterogeneous reactions to such common shocks. For an excellent review, see Sarafidis and Wansbeek (2012). A range of estimators are available in the literature. For example, Pesaran (2006) proposed the common correlated effects (CCE) estimator, which does not require estimating the number of unobserved common factors and tries to eliminate the effects of the common factor. Bai (2009) proposed an iterative principle component (IPC) estimator. Both Pesaran's CCE estimator and Bai's IPC estimator require $N$ and $T$ to jointly go to infinity so that consistency and asymptotic normality are achievable.

In this study, we aim to (1) identify and quantify the heterogeneous impacts of both observable factors and unobservable common shocks on stock price movements; and (2) identify companies within a particular industry that are more sensitive to industry-wide common shocks than others. Besides applying existing econometric methods in the literature, we propose a new Bayesian estimator for factor structure models. The Bayesian algorithm, using Gibbs sampling with data augmentation, makes estimating the common shocks at each time period, as well as the reactions of each individual company to these common shocks, more straightforward. Estimating these unobservable common factors and factor loadings of each company allows us to investigate the sensitivity of stock returns to commonly shared unobservable shocks, which has significant implications for the proper valuation of companies. The sensitivity of a stock return to unobserved common shocks could indicate how prone the company stock is to industry-wide trend or cyclicality. As companies in the 
same industry are often direct competitors, investigating the sensitivity of the industry would enhance our ability to understand the industry-wide competitive relationship.

The rest of this paper is organized as follows. In Section 2 we review the empirical model, i.e. the equity valuation model, and generalize it to take into account of panel data structure. Section 3 discusses the data issues and conducts formal CSD tests for our data. In Section 4, we propose a Bayesian estimator for panel data model with factor structural error terms. Section 5 presents results from different models. Section 6 summarizes and concludes the paper.

\section{Fundamental Equity Valuation Model}

In order to capture the company-specific factors that influence stock returns, we employ the equity valuation model of Zhang (2000). Chen and Zhang (2007) extended the model to establish the theoretical relationship between stock returns and accounting fundamentals. The model measures the characteristics of underlying operations of a company using the links between the future cash flows and observed accounting data in valuing equity. Equity value is a function of two basic operational attributes: scale and profitability.

Let $V_{t}$ be the value of an all-equity finance company at date $t$. The variable $V_{t}$ represents the present value of future cash flows, hence the equity value of a firm. We are interested in the equity value of a firm, using accounting information; hence model the value of a firm, net of any debts. $B_{t}$ is the corresponding book value of equity. $X_{t}$ is the earnings generated in period $t$, and $g_{t}$ is the company's growth opportunities as perceived at $t . g_{t}$ is defined as the percentage by which capital invested may grow. The variable $r_{t}$ is discount rate at $t$. Let $q_{t} \equiv X_{t} / B_{t-1}$ be profitability at time $t$. Let $E_{t}\left(X_{t+1}\right)$ be the expected next period earnings, $k$ is the earnings capitalization factor, and $P\left(q_{t}\right)$ and $C\left(q_{t}\right)$ are the put option to abandon operations and the call option to expand operations, respectively. $P\left(q_{t}\right)$ and $C\left(q_{t}\right)$ are normalized by the book value, $B_{t}$. To simplify the analysis, assume that profitability follows a random walk, $\tilde{q}_{t+1}=q_{t}+\tilde{e}_{t+1}$. Chen and Zhang (2007) derived the valuation function of equity as

$$
V_{t}=B_{t}\left[q_{t} / r_{t}+P\left(q_{t}\right)+g_{t} C\left(q_{t}\right)\right] \equiv B_{t} v\left(q_{t}, g_{t}, r_{t}\right)
$$

where $v\left(q_{t}, g_{t}, r_{t}\right) \equiv q_{t} / r_{t}+P\left(q_{t}\right)+g_{t} C\left(q_{t}\right)$. Now consider $\Delta V_{t+1}$, the change in equity value from date $t$ to date $t+1$. Define $v_{1} \equiv \mathrm{d} v / \mathrm{d} q_{t}$ and $v_{3} \equiv \mathrm{d} v / \mathrm{d} r_{t} . \mathrm{d} v / \mathrm{d} g_{t}$ is $E\left(q_{t}\right)$ and need not be defined again. Let $D_{t}$ be the dividends paid in period $t+1$. Chen and Zhang (2007) derived the 
period $t+1$ stock return, denoted $R_{t+1}$ as

$$
R_{t+1}=\frac{X_{t+1}}{V_{t}}+v_{1} \frac{B_{t}}{V_{t}} \Delta q_{t+1}+\left(1-\frac{B_{t}}{V_{t}}\right) \frac{\Delta B_{t+1}}{B_{t}}+C\left(q_{t}\right) \frac{B_{t}}{V_{t}} \Delta g_{t+1}+v_{1} \frac{B_{t}}{V_{t}} \Delta r_{t+1} .
$$

For more details of this valuation model, please see Chen and Zhang (2007). Based on (2), Chen and Zhang (2007) ran the following approximated regression.

$$
R_{i t}=\alpha+\beta x_{i t}+\gamma \Delta \hat{q}_{i t}+\delta \Delta \hat{b}_{i t}+\omega \Delta \hat{g}_{i t}+\phi \Delta \hat{r}_{i t}+e_{i t},
$$

where $R_{i t}$ is the annual stock return; $x_{i t}=X_{i t} / V_{i t-1}$ is the earnings yield, divided by the beginning-of-period market value of equity; $\Delta \hat{q}_{i t}=\left(q_{i t}-q_{i t-1}\right) B_{i t-1} / V_{i t-1}$ is the change in profitability, adjusted by the beginning-of-period ratio of the book value of equity to the market value of equity, with profitability defined as the return on equity; $\Delta \hat{b}_{i t}=\left[\left(B_{i t}-B_{i t-1}\right) / B_{i t-1}\right]\left(1-B_{i t-1} / V_{i t-1}\right)$ is capital investment, adjusted by one minus the beginning-of-period book-to-market ratio; $\Delta \hat{g}_{i t}=\left(g_{i t}-g_{i t-1}\right) B_{i t-1} / V_{i t-1}$ is the change in growth opportunities, adjusted by the beginning-of-period book-to-market ratio; $\Delta \hat{r}_{i t}=\left(r_{t}-r_{t-1}\right) B_{i t-1} / V_{i t-1}$ is the change in the discount rate, adjusted by company's beginningof-period book-to-market ratio; and $e_{i t}$ 's are random error terms and independent of each other.

Growth opportunities, $g$, are often predicted using long-term analysts' forecasts. The availability of these forecasts for an individual company is severely limited. To avoid loss of observations, we drop $g$ from our empirical model, and take the four accounting variables as our fundamental variables. We adopt S\&P500 index as a proxy for market-wide common shocks, and the empirical model is:

$$
R_{i t}=\alpha+\beta x_{i t}+\gamma \Delta \hat{q}_{i t}+\delta \Delta \hat{b}_{i t}+\phi \Delta \hat{r}_{i t}+\zeta S P 500_{t}+e_{i t} .
$$

This model specification takes a form of arbitrage pricing theory (APT) and includes a stock market return as an explanatory variable. Therefore, it could be seen as an extension of Capital Asset Pricing Model (CAPM). However, equation (4) is different from CAPM in that the market return is not the only factor that explains the dependent variable. It is closer to a multifactor explanatory model, which extends that of Chen and Zhang (2007). The ex-ante expectation of the signs of $\beta$ is positive in general as stock return would decrease when earnings decrease. Therefore, if there is a dramatic decrease in earnings, stock return is expected to drop significantly. We expect $\gamma$ to be positive in general because change in profitability is positively related to stock price movements. The sign of $\phi$ is expected to be 
negative, as increase in the discount rate would reduce the future cash flow of a company. In our empirical analysis, we further allow coefficients in Equation (4) to vary across individual companies to capture heterogeneous impacts of observed factors; and $e_{i t}{ }^{\prime} s$ to be correlated across individuals, i.e. $\operatorname{cov}\left(e_{i t}, e_{j t}\right) \neq 0$ for $i \neq j$, to allow for CSD.

\section{Data and Preliminary Tests}

\subsection{Data Description and Data Issues}

Excluding industries that have less than three ${ }^{1}$ companies and companies that do not have data available for the entire sample period, our analyses are based on the monthly returns of 56 stocks across six industries in the S\&P100 index between January 2003 and December 2012. In particular, we have 11 companies from the IT industry, 13 from the industrial industry, 8 from the energy industry, 10 from the financial industry, 11 from the health care industry and 3 from the utilities industry. Stock prices are sourced from Bloomberg. The company-level accounting data is available from the Compustat North America database and Thomson Reuters' Worldscope database. We use analyst long-term forecasts from the Institutional Broker Estimate System (IBES). We follow the approach of Hong and $\mathrm{Wu}$ (2014) in our sample construction except that we do not adopt portfolios for our analyses. We assume that companies from the same industries are more likely to be correlated with each other due to unobserved industry-wide common shocks (e.g. news or policies) compared to companies from different industries. Therefore we conduct the analysis for each industry separately.

Many existing studies of using accounting information to explain stock returns become event studies as they collected stock returns on or around the earnings announcement date (see Chen and Zhang, 2007; Clement et al., 2011). As our main interest is in the industrywide factors, we use monthly stock return data based on calendar dates. Although this yields much lower statistical significance for the estimated coefficients of the accounting variables, such structure allows us to overcome the restrictions of the event study framework.

Mixed-frequency problem is another issue in our dataset, since accounting data is produced quarterly, while stock price data is produced monthly. In order to overcome this, we follow the method of Hong and Wu (2014). There are two different types of data in our sample: stock and flow. Stock data is snapshots of the measured variable at a given point in

1 At least three companies are needed to identify one industry-wide common factor. Details are discussed in Section 4. 
time, whereas flow data represents an accumulation over a given period. Stock return, profitability, growth opportunity and discount rate are stock variables, but earnings, yield and capital investments are flow variables. By accumulating monthly observations of flow variables over a quarter, they could then become the end of the quarter observation. This means the end of quarter observation for flow variables could be, at least in theory, reverse engineered and decomposed into monthly observations. Since all of our quarterly observed variables are flow variables, weighted average is used under this assumption.

Such reverse engineering allows us to only investigate ex-post explanatory power of accounting fundamentals, observed market-wide common shocks and unobserved industrywide common shocks. This is consistent with the focus of the paper, i.e. the contemporaneous relationship between stock returns and the given explanatory factors. Investigation of the contemporaneous regression analysis also has another benefit of being consistent to the existing literature including Zhang (2000) and Chen and Zhang (2007).

\subsection{Cross-Sectional Dependence Test and Number of Common Factors}

We first conduct a formal cross-sectional dependence (CSD) test to our data set to see if CSD exists. For each industry, our panel data set has small $N$ and relatively large $T$, therefore we adopt the CSD test proposed by Breusch and Pagan (1980). This test is based on the following Lagrange multiplier (LM) statistic

$$
C S D_{L M}=T \sum_{i=1}^{N-1} \sum_{j=i+1}^{N} \hat{\rho}_{i j}^{2},
$$

where $\hat{\rho}_{i j}$ is the sample estimated pair-wide correlation of the residuals. Specifically,

$$
\hat{\rho}_{i j}=\hat{\rho}_{j i}=\frac{\sum_{t=1}^{T} \hat{e}_{i t} \hat{e}_{j t}}{\left(\sum_{t=1}^{T} \hat{e}_{i t}^{2}\right)^{1 / 2}\left(\sum_{t=1}^{T} \hat{e}_{j t}^{2}\right)^{1 / 2}},
$$

where $\hat{e}_{i t}$ is the estimate of $e_{i t}$ in equation (4) by running OLS regression for each individual $i$. Breusch and Pagan (1980) show that under the null hypothesis of CSD, $C S D_{L M}$ is asymptotically a chi-squared distribution with $N(N-1) / 2$ degrees of freedom.

(Insert Table 1 here)

We conduct the CSD test for each of the six industries, with Table 1 presenting the degree of freedom, the LM statistic of $C S D_{L M}$, and the corresponding $p$-value for each 
industry. The test results indicate that CSD exists in all industries.

\section{Econometric Frameworks}

There are a range of estimators proposed to take into account CSD in the literature. For an excellent review, see Sarafidis and Wansbeek (2012). SUR approach, due to Zellner (1962), is the most classical econometric model of CSD for panel data with small $N$ and large $T$. Pesaran (2006) proposed the common correlated effects (CCE) estimator and Bai (2009) introduced the iterative principal components (IPC) estimator. For both the CCE and IPC estimators, large $N$ and $T$ are required. This requirement implies that applying these two methods to capture industry-wide common shocks may not perform well, given we only have a small number of companies within each industry.

In this study we propose a new Bayesian estimator to allow for CSD in panel data with large $T$ and small $N$, which is specifically suitable to capture the impacts of unobserved industry-wide common shocks on stock price movements. For each industry, we specify a factor structure model as

$$
\underset{1 \times 1}{y_{i t}}=\mathbf{z}_{i t}^{\prime} \underset{s \times 1}{\boldsymbol{\theta}_{i}}+e_{i t} \text {, where } \underset{1 \times 1}{e_{i t}}=\boldsymbol{\lambda}_{i}^{\prime} \underset{m \times 1}{\boldsymbol{f}_{t}}+\varepsilon_{i t} .
$$

So it allows for heterogeneous impacts of observed regressors through individual specific $\boldsymbol{\theta}_{i}$ and CSD through factor structure error terms. Note that same to other panel data models with multifactor structure error terms, imposing such structure causes loss of unrestricted error covariance matrix in the SUR model. However, it allows us to see how companies respond to the industry-wide common shocks heterogeneously. Model in Equation (7) is a generalization of that proposed by Geweke and Zhou (1996) through introducing z regressors.

For the sake of identification, it is required that $m \leq(N-1) / 2$ (Geweke and Zhou, 1996, p. 565). For simplicity, we write the model in matrix form as

$$
\underset{N \times 1}{\boldsymbol{Y}_{t}}=\underset{N \times N s}{\boldsymbol{Z}} \underset{N s \times 1}{\boldsymbol{\theta}}+\underset{N \times m}{\boldsymbol{\Lambda}} \underset{m \times 1}{\boldsymbol{f}_{t}}+\underset{N \times 1}{\boldsymbol{\varepsilon}_{t}},
$$

where

$$
\boldsymbol{Y}_{t}=\left(\begin{array}{c}
y_{1 t} \\
\vdots \\
y_{N t}
\end{array}\right), \quad \boldsymbol{Z}_{t}=\left(\begin{array}{ccc}
\mathbf{z}_{1 t}^{\prime} & & \\
1 \times s & & \\
& \ddots & \\
& & \mathbf{z}_{N t}^{\prime} \\
1 \times s
\end{array}\right), \quad \boldsymbol{\theta}=\left(\begin{array}{c}
\boldsymbol{\theta}_{1} \\
\vdots \\
\boldsymbol{\theta}_{N}
\end{array}\right), \quad \Lambda=\left(\begin{array}{c}
\lambda_{1}^{\prime} \\
\vdots \\
\lambda_{N}^{\prime}
\end{array}\right) \quad \text { and } \quad \boldsymbol{\varepsilon}_{t}=\left(\begin{array}{c}
\varepsilon_{1 t} \\
\vdots \\
\varepsilon_{N t}
\end{array}\right) \text {. }
$$

Then the following conditions are standard and necessary for the Bayesian analysis (Geweke and Zhou, 1996; Chan et al., 2013). 
Assumption 1: The error terms and factors are independent and identically distributed across t. Specifically,

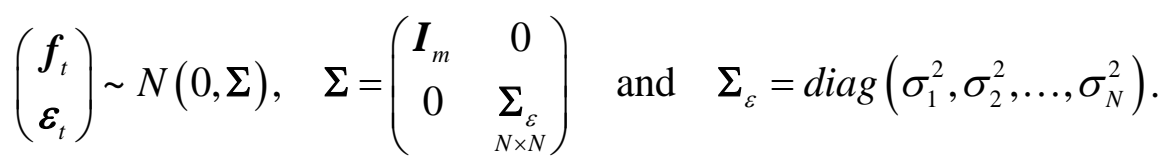

Moreover, $\boldsymbol{f}_{t}$ and $\boldsymbol{\varepsilon}_{t}$ are independent of $\boldsymbol{Z}_{t}$.

Follow the spirit of the Bayesian algorithm presented in Geweke and Zhou (1996) we propose a Bayesian Gibbs sampler for the model of (8). Both $\lambda_{i}$ 's and $\boldsymbol{f}_{t}$ 's are treated as unknown parameters with the values being drawn from their conditional posterior densities. Then, conditional on $\lambda_{i}$ 's and $\boldsymbol{f}_{t}$ 's, the model of (8) reduces to the standard linear regression model, facilitating draws from the conditional posterior densities for the parameters. Note that the above set-up rules out the correlation between regressors and factors (or regressors and factor loadings), which is a drawback of this study.

For prior densities of individual-varying slope coefficients and variances of random error terms, we consider a standard diffuse prior, that $\boldsymbol{\theta}_{i} \propto \mathbf{1}_{s}$ and $h_{i} \propto \frac{1}{h_{i}}$, where $h_{i}=\frac{1}{\sigma_{i}^{2}}>0$. For prior densities of unobservable factor loadings, we follow Geweke and Zhou (1996) to have $\lambda_{i} \propto \mathbf{1}_{m}$ for the case with large $T$ and small $N$. Under these prior distributions and model specifications, the joint posterior density for all unknown parameters (such that $\boldsymbol{\theta}_{i}$, $h_{i}, \quad \lambda_{i}$ and $\boldsymbol{f}_{t}$ ) can be written as

$$
\begin{aligned}
p\left(\Theta \mid \boldsymbol{Y}_{1: T}, \boldsymbol{Z}_{1: T}\right)= & \prod_{i=1}^{N} \prod_{t=1}^{T}(2 \pi)^{-1 / 2} h_{i}^{1 / 2} \exp \left\{-\frac{h_{i}}{2}\left(y_{i t}-\boldsymbol{z}_{i t}^{\prime} \boldsymbol{\theta}_{i}-\lambda_{i}^{\prime} \boldsymbol{f}_{t}\right)^{2}\right\} \\
& \cdot \prod_{i=1}^{N} \frac{1}{h_{i}} \cdot \prod_{t=1}^{T}(2 \pi)^{-m / 2} \exp \left\{-\frac{1}{2} \boldsymbol{f}_{t}^{\prime} \boldsymbol{f}_{t}\right\},
\end{aligned}
$$

where $\Theta$ represents for all unknown parameters.

From the joint posterior density (9) we can derive the full conditional posterior densities that can be used for Gibbs sampling. Particularly, we assume that all the rank conditions needed below are satisfied.

The conditional posterior densities of the parameter $\theta_{i}$ 's for $i=1, \ldots, N$ are normal distributions, given by

$$
\boldsymbol{\theta}_{i} \mid \boldsymbol{Y}_{1: T}, \boldsymbol{Z}_{1: T}, \Theta_{-\boldsymbol{\theta}_{i}} \sim N\left(\hat{\boldsymbol{\theta}}_{i}, \frac{1}{h_{i}}\left(\mathbf{Z}_{i}^{\prime} \mathbf{Z}_{i}\right)^{-1}\right),
$$


where

$$
\hat{\boldsymbol{\theta}}_{i}=\left(\boldsymbol{Z}_{i}^{\prime} \boldsymbol{Z}_{i}\right)^{-1}\left(\boldsymbol{Z}_{i}^{\prime} \tilde{\boldsymbol{Y}}_{i}\right), \quad \underset{T \times S}{\boldsymbol{Z}_{i}}=\left(\begin{array}{c}
\mathbf{z}_{i 1}^{\prime} \\
\vdots \\
\boldsymbol{z}_{i T}^{\prime}
\end{array}\right) \text { and } \quad \tilde{\boldsymbol{Y}}_{T \times 1}=\left(\begin{array}{c}
y_{i 1}-\lambda_{i}^{\prime} \boldsymbol{f}_{1} \\
\vdots \\
y_{i T}-\lambda_{i}^{\prime} \boldsymbol{f}_{T}
\end{array}\right) \text {. }
$$

The conditional posterior densities of $h_{i}$ 's for $i=1, \ldots, N$ are gamma distributions as follows.

$$
h_{i} \mid \boldsymbol{Y}_{1: T}, \boldsymbol{Z}_{1: T}, \boldsymbol{\Theta}_{-h_{i}} \sim G\left(s_{h}, s_{c}\right) \text {, }
$$

where the shape and scale parameters are $s_{h}=\frac{T}{2}$ and $s_{c}=2 / \sum_{t=1}^{T}\left(y_{i t}-z_{i t}^{\prime} \boldsymbol{\theta}_{i}-\lambda_{i}^{\prime} \boldsymbol{f}_{t}\right)^{2}$ respectively. Specifically, if $z \sim G\left(s_{h}, s_{c}\right)$, then $p\left(z \mid s_{h}, s_{c}\right)=\frac{s_{c}{ }^{-s_{h}} z^{s_{h}-1}}{\Gamma\left(s_{h}\right)} \exp \left(-z / s_{c}\right)$.

The conditional posterior densities of $\boldsymbol{f}_{t}$ 's for $t=1, \ldots, T$ are normal distributions, given by

$$
\boldsymbol{f}_{t} \mid Y_{1: T}, Z_{1: T}, \Theta_{-f_{t}} \sim N\left(\hat{\boldsymbol{f}}_{t},\left(I_{m}+\tilde{\Lambda}^{\prime} \tilde{\Lambda}\right)^{-1}\right)
$$

where

$$
\hat{\boldsymbol{f}}_{t}=\left(I_{m}+\tilde{\Lambda}^{\prime} \tilde{\Lambda}\right)^{-1} \tilde{\Lambda}^{\prime} \tilde{Y}_{t}, \quad \tilde{Y}_{t}=\left(\begin{array}{c}
h_{1}^{1 / 2} \cdot\left(y_{1 t}-\mathbf{z}_{1 t}^{\prime} \boldsymbol{\theta}_{1}\right) \\
\vdots \\
h_{N}^{1 / 2} \cdot\left(y_{N t}-z_{N t}^{\prime} \boldsymbol{\theta}_{N}\right)
\end{array}\right), \quad \tilde{\Lambda}=\left(\begin{array}{c}
h_{1}^{1 / 2} \cdot \lambda_{1}^{\prime} \\
\vdots \\
h_{N}^{1 / 2} \cdot \lambda_{N}^{\prime}
\end{array}\right)
$$

For the purpose of identification (Geweke and Zhou, 1996), we set the first $m$ rows of $\Lambda=\left(\begin{array}{c}\Lambda_{1} \\ m \times m \\ \Lambda_{2} \\ (N-m) \times m\end{array}\right)$ as

$$
\Lambda_{1}=\left(\begin{array}{cccc}
\lambda_{11} & 0 & \cdots & 0 \\
\lambda_{21} & \lambda_{22} & & 0 \\
\vdots & \vdots & \ddots & \vdots \\
\lambda_{m 1} & \lambda_{m 2} & \cdots & \lambda_{m m}
\end{array}\right) \text { and } \lambda_{i i}>0 \text { for } i=1, \ldots, m
$$

Thus, for the first $m$ factor loadings

$$
\lambda_{i} \mid Y_{1: T}, Z_{1: T}, \Theta_{-\gamma_{i}} \sim N\left(\hat{\lambda}_{i}, \frac{1}{h_{i}}\left(F_{i}^{\prime} F_{i}\right)^{-1}\right) \text {, }
$$


where $\quad \hat{\lambda}_{i}=\left(F_{i}^{\prime} F_{i}\right)^{-1} F_{i} \hat{Y}_{i}, \quad \underset{T \times i}{F_{i}}=\left(\begin{array}{c}\boldsymbol{f}_{1 i}^{\prime} \\ \vdots \\ \boldsymbol{f}_{T i}^{\prime}\end{array}\right), \quad \boldsymbol{f}_{i \neq 1}=\left(\begin{array}{c}f_{t, 1} \\ \vdots \\ f_{t, i}\end{array}\right)$ and $\hat{Y}_{i}=\left(\begin{array}{c}y_{i 1}-\mathbf{z}_{i 1}^{\prime} \boldsymbol{\theta}_{i} \\ \vdots \\ y_{i T}-\mathbf{z}_{i T}^{\prime} \boldsymbol{\theta}_{i}\end{array}\right), \quad$ for $i=1, \ldots, m$. For the rest factor loadings $(i=m+1, \ldots, N)$,

$$
\lambda_{i} \mid Y_{1: T}, Z_{1: T}, \Theta_{-\gamma_{i}} \sim N\left(\hat{\lambda}_{i}, \frac{1}{h_{i}}\left(F^{\prime} F\right)^{-1}\right)
$$

where $\hat{\lambda}_{i}=\left(F^{\prime} F\right)^{-1} F^{\prime} \hat{Y}_{i}, \quad F=\left(\begin{array}{c}\boldsymbol{f}_{1}^{\prime} \\ \vdots \\ \boldsymbol{f}_{T}^{\prime}\end{array}\right)$ and $\hat{Y}_{i}=\left(\begin{array}{c}y_{i 1}-\mathbf{z}_{i 1}{ }^{\prime} \boldsymbol{\theta}_{i} \\ \vdots \\ y_{i T}-\mathbf{z}_{i T}{ }^{\prime} \boldsymbol{\theta}_{i}\end{array}\right)$.

\section{Empirical Results}

To capture the impact of industry-wide common shocks on stock price movements, we apply both the SUR approach and our Bayesian estimator to each industry. Since both Pesaran's (2006) CCE estimators and Bai's (2009) IPC estimator require large $N$ and large $T$, they are not suitable to capture industry-wide common shocks given that we only have relatively a small number of companies in each industry. Alternatively we can pool all companies together regardless of industry classification, then the CCE estimator and IPC estimator can be applied to account for more general market-wide unobserved common shocks. However stock returns of companies from the same industries are more likely to be correlated with each other, compared to those from different industries. Therefore in this study, we focus on applying the two former methods, i.e. the SUR approach and the Bayesian estimator, to capture industry-wide common shocks. Results for pooled data from all four methods without discussion are presented in Appendix for the sake of space limitation.

\subsection{Impact of Observable Factors on Stock Returns}

We first estimate factor structure model (i.e. (7)) using our Bayesian estimator separately for all six industries. As stated in Section 4, the highest number of common factors that can be identified is less than half of the number of individual companies. Since there are only three companies in the industry of utilities, only one common factor can be identified. For the sake of comparison, we first set the number of common factors as one for all six industries. For each model, we generate 15,000 draws from the posterior conditional distributions and discard the first 5,000 as a "burn-in".

(Insert Table 2 here) 
Due to space limitations, we present the estimated coefficients for IT industry ${ }^{2}$ only. Table 2 presents the posterior statistics, including posterior mean, standard deviation and 95 per cent credible interval (CI) for coefficients of observable accounting variables and the S\&P500 index, proxy for market-wide common shocks, for each company from the IT industry. In order to assess the Gibbs sampler's performance, we present the sampled paths and auto-correlation functions (ACFs) of these sample paths for all estimated coefficients of Apple Inc. in Figure 1. These plots show that the sample paths are mixed well. In particular, the time series sample paths for all estimated parameters are randomly distributed within a small range without any time trend. Second, the auto-correlation plots for all parameters decrease to zero rapidly within 10 draws. The sampled paths and ACF's of estimated coefficients for the other companies are very similar, and are not included here.

\section{(Insert Figure 1 here)}

In general, we find that market-wide common shock, represented by the S\&P500 index, has statistically significant positive impacts, with none of the 95 per cent CIs containing zero, on stock returns. On the other hand, most CIs of estimated coefficients for accounting fundamentals include zero, indicating no significant impact from accounting fundamentals on stock returns. In addition, we find that the impacts of observed market-wide common shock and accounting fundamentals on stock returns vary across companies. For example, the impact of market S\&P500 index, varies across companies from 0.7510 (for IBM) to 1.3613 (for Dell). The adjusted capital investment, $\Delta b$, only has significantly positive impact on stock returns for three companies: Apple Inc., Intel Corporation and Qualcomm Inc., but has no significant impact for the other eight IT companies. Furthermore, the magnitudes of such significant impacts are different, ranging from 1.5060 (for Intel Corporation) to 2.0254 (for Apple Inc.). Therefore, the model specification of individual-varying coefficients of observable accounting fundamentals and market-wide common factors can be justified.

For model comparison, we also estimate SUR models for all industries separately. Results for all companies from the IT industry are presented in Table $3^{3}$. We observe three major findings by comparing the estimated results from SUR model and Bayesian common factor model.

\footnotetext{
${ }^{2}$ Results for all the other industries have consistent implications to those for IT industry. They are ava ilable from the authors upon request.

${ }^{3}$ SUR models are estimated by the feasible GLS (FGLS) method rather than Bayesian MCMC method. Results for other industries are available upon request.
} 
(Insert Table 3 here)

First, the results from SUR models show that almost all of the account fundamentals, except for the change in profitability, have significant impacts on IT companies' stock returns. In particular, the earnings yield $(x)$ has a significant affect on stock returns for eight out of eleven IT companies. The impact of capital investment $(\Delta b)$ is significant for all IT companies except for EMC Corporation. Eight IT companies' stock returns are significantly impacted by the change in the discount rate $(\Delta r)$. But such significant impacts almost all disappear, when the unobserved industry-wide factor is taken into account, as by the Bayesian common factor model. However, it is worthwhile to note that the point estimations by the SUR model are very close to the posterior mean estimations for any single parameters of accounting fundamentals.

Second, estimated impacts of S\&P500 index on stock returns by the SUR model and those by Bayesian common factor model are almost identical. Therefore, the explaining power of S\&P500 index to stock price movement is robust.

Third, results from the SUR model also show considerable variations in estimated coefficients across companies, which demonstrates that there exists strong individual heterogeneity within the IT industry. For example, the estimated impacts of S\&P500 index on stock returns range from 0.7433 (for IBM) to 1.3732 (for Dell). The significant impacts of earnings yield $(x)$ on stock returns have an even higher range, from -1.9016 (for Qualcomm Inc.) to 0.5332 (for Accenture Plc.)

\subsection{Impact of Unobservable Common Shocks on Stock Returns}

In this section, we first compare the explaining power of observed factors, i.e. accounting fundamentals and market-wide common factor, versus that of unobserved common shocks in the Bayesian common factor model. In doing so, we calculate two variance ratios indicating explaining power of observed factors and unobserved common shocks, respectively. In particular the explaining power of observed factors can be expressed as $\operatorname{Var}\left(\mathbf{z}_{i t}^{\prime} \hat{\boldsymbol{\theta}}_{i}\right) / \operatorname{Var}\left(y_{i t}\right)$, and that of unobserved common shocks is $\operatorname{Var}\left(\hat{\lambda}_{i} \hat{\boldsymbol{f}}_{t}\right) / \operatorname{Var}\left(y_{i t}\right)$, given that it is assumed $\boldsymbol{f}_{t}$ is independent of $\mathbf{z}_{i t}$. Table 4 presents these two variance ratios for each IT companies.

\section{(Insert Table 4 here)}

Two findings emerge from these results. First, we can see that unobserved common shocks explain considerable proportions of variation in stock returns for IT companies, after 
controlling the impacts from observed factors. For example, on individual basis, unobserved common shocks can explain more than $15 \%$ of variation in stock returns for EMC Corporation. More than $6 \%$ of the overall variation in stock returns in the IT industry can be attributed to the unobserved common shocks within this industry. Second, the extents to which each IT company was exposed to unobserved industry-wide common shocks are different. That is to say, some companies are more sensitive to industry-wide common shocks than other companies. For example, the results show that the most sensitive company is EMC Corporation with $15.7 \%$ variation in stock returns attributed to occurrence of industry-wide common shocks, while the least sensitive company is Microsoft with only around $1 \%$ variation in its stock returns can be explained by unobserved common shocks.

\subsection{Robustness Check: Number of Common Factors}

So far we set the number of unobserved industry-wide common factors as one because only one common factor can be identified for the industry of utilities. To check the robustness of this assumption for the other five industries (IT, industrial, energy, finance and health care), we estimate the Bayesian common factor models with two and three ${ }^{4}$ common factors, respectively, for each of the five industries. To show how well each model specification fits the data, we report the overall Mean Squared Errors (MSE's) estimated by Bayesian factor models with the specification of one, two or three common factors for each of the five industries. Specifically, the MSE can be calculated as

$$
\operatorname{MSE}=\frac{1}{N T} \sum_{i=1}^{N} \sum_{t=1}^{T}\left(y_{i t}-\hat{y}_{i t}\right)^{2},
$$

where $\hat{y}_{i t}$ are the stock returns estimated by Bayesian common factor model, i.e. $\hat{y}_{i t}=\mathbf{z}_{i t}^{\prime} \hat{\boldsymbol{\theta}}_{i}+\hat{\lambda}_{i}^{\prime} \hat{\boldsymbol{f}}_{t}$.

\section{(Insert Table 5 here)}

Table 5 reports MSE's from each Bayesian common factor model for each of the five industries. The results show that increasing the number of common shocks cannot make Bayesian common factor models fit the data in any significantly better way. In particular, for the IT industry, an increase in the number of common shocks has almost no impact on MSE of the model. For the other four industries, there is a minor improvement in MSE when the

\footnotetext{
${ }^{4}$ Three is the highest number of common factors that can be identified for the industry of energy.
} 
number of common shocks is increased from one to two, such as a decrease in MSE from 0.0030 to 0.0026 for the industrial industry. Increasing the number of common factors from two to three has almost no impact, and in some industries, such as energy, MSE is even increased. These results imply that our specification of one common factor is reasonable, and that increasing the number of shocks does not dramatically improve the model's performance. It is worthwhile to note that we are not using MSE as a criterion for model selection in terms of specification on the number of common factors. In Bayesian framework, such model selection is still a research area requiring further study and is out of the scope of this paper.

\section{Conclusion}

This paper applies two different econometric models, i.e. SUR approach and a Bayesian estimator for panel data regression model with multi-factor structural error terms, to examine the causes of stock price movements. Differing from previous methods applied in this literature, these two methods allow for cross-sectional dependence existing among companies from the same industry, caused by unobserved industry-wide common shocks. In the factor structure model, we assume that the industry-wide common shocks impact on all companies in the industry but with different intensities. We propose a new Bayesian estimator specifically for the factor structure model for panel data with small $N$ and large $T$.

The empirical results from this study show that the impacts of observed accounting fundamentals and market-wide common shocks are heterogeneous across companies within the same industry. That is to say, the same factor has very different impacts on stock returns for different companies. Market-wide common shocks have stronger and more significant impacts on stock returns compared to accounting fundamentals. After controlling observed accounting fundamentals and market-wide common factors, considerable proportions of variations in stock returns can be attributed to industry-wide common shocks. We also find reactions of different companies to unobserved industry-wide common shocks are different. Some companies' stock returns are more sensitive to industry-wide common shocks than others'. 
Table 1 Breusch and Pagan's Test of Cross-Section Dependence

\begin{tabular}{lrrrrrr}
\hline & IT & Industrial & Energy & Finance & Health Care & Utilities \\
Degree freedom & 55 & 78 & 28 & 45 & 55 & 3 \\
$C S D_{L M}$ & 183 & 234 & 371 & 441 & 346 & 55 \\
$p$-value & 0.00 & 0.00 & 0.00 & 0.00 & 0.00 & 0.00 \\
\hline
\end{tabular}


Table 2 Estimated Coefficients of Observed Factors for the IT Industry from Bayesian Common Factor Model

\begin{tabular}{|c|c|c|c|c|c|c|c|}
\hline \multicolumn{4}{|c|}{ Apple Inc. } & \multicolumn{4}{|c|}{ Accenture Plc. } \\
\hline & Mean & Std & $95 \%$ CI & & Mean & Std & $95 \%$ CI \\
\hline $\mathrm{x}$ & -1.6936 & 1.7674 & $(-5.1657,1.7444)$ & $\mathrm{x}$ & 0.4542 & 0.3473 & $(-0.2254,1.1313)$ \\
\hline$\Delta \mathrm{q}$ & -4.6425 & 6.5265 & $(-17.579,8.3076)$ & $\Delta q$ & -0.7904 & 3.3818 & $(-7.3441,5.7851)$ \\
\hline$\Delta \mathrm{b}$ & 2.0254 & 0.3939 & $(1.2574,2.7924)$ & $\Delta \mathrm{b}$ & -0.0217 & 0.0924 & $(-0.2028,0.1577)$ \\
\hline$\Delta \mathrm{r}$ & 0.1426 & 0.2886 & $(-0.4243,0.7073)$ & $\Delta \mathrm{r}$ & -0.0871 & 0.2839 & $(-0.6469,0.4608)$ \\
\hline SP500 & 1.2218 & 0.2237 & $(0.7758,1.6576)$ & SP500 & 0.9257 & 0.1144 & $(0.7001,1.1511)$ \\
\hline \multicolumn{4}{|c|}{ Cisco System } & \multicolumn{4}{|c|}{$\underline{\text { Dell }}$} \\
\hline & Mean & Std & $95 \%$ CI & & Mean & Std & $95 \%$ CI \\
\hline $\mathrm{x}$ & -0.3379 & 0.4660 & $(-1.2696,0.5722)$ & $\mathrm{x}$ & -0.8304 & 0.7502 & $(-2.3010,0.6343)$ \\
\hline$\Delta q$ & 10.3445 & 6.7426 & $(-2.8156,23.708)$ & $\Delta \mathrm{q}$ & -0.8990 & 3.9800 & $(-8.6342,6.9031)$ \\
\hline$\Delta \mathrm{b}$ & -0.0902 & 0.1559 & $(-0.3958,0.2159)$ & $\Delta \mathrm{b}$ & -0.0994 & 0.1209 & $(-0.3406,0.1398)$ \\
\hline$\Delta \mathrm{r}$ & -0.1777 & 0.1384 & $(-0.4508,0.0946)$ & $\Delta \mathrm{r}$ & -0.0868 & 0.3423 & $(-0.7469,0.5861)$ \\
\hline SP500 & 1.3435 & 0.1367 & $(1.0779,1.6098)$ & SP500 & 1.3613 & 0.1642 & $(1.0379,1.6801)$ \\
\hline \multicolumn{4}{|c|}{$\underline{\text { EMC Corporation }}$} & \multicolumn{4}{|c|}{ Hewlett Packard } \\
\hline & Mean & Std & $95 \%$ CI & & Mean & Std & $95 \%$ CI \\
\hline $\mathrm{x}$ & 0.3083 & 1.0673 & $(-1.7994,2.4126)$ & $\mathrm{x}$ & 0.2591 & 0.2746 & $(-0.2703,0.8001)$ \\
\hline$\Delta q$ & -3.2525 & 5.9631 & $(-14.793,8.4963)$ & $\Delta q$ & 0.6026 & 0.3785 & $(-0.1338,1.3421)$ \\
\hline$\Delta \mathrm{b}$ & -0.0840 & 0.3460 & $(-0.7604,0.5939)$ & $\Delta \mathrm{b}$ & -0.3546 & 0.2698 & $(-0.8817,0.1792)$ \\
\hline$\Delta \mathrm{r}$ & -0.0667 & 0.0969 & $(-0.2554,0.1228)$ & $\Delta \mathrm{r}$ & -0.0302 & 0.0865 & $(-0.1972,0.1396)$ \\
\hline SP500 & 1.3198 & 0.1561 & $(1.0108,1.6270)$ & SP500 & 1.1709 & 0.1343 & $(0.9072,1.4375)$ \\
\hline \multicolumn{4}{|c|}{$\underline{\mathrm{IBM}}$} & \multicolumn{4}{|c|}{$\underline{\text { Intel Corporation }}$} \\
\hline & Mean & Std & $95 \%$ CI & & Mean & Std & $95 \%$ CI \\
\hline $\mathrm{x}$ & 0.3211 & 0.3658 & $(-0.4112,1.0421)$ & $\mathrm{x}$ & -0.5264 & 0.4407 & $(-1.3925,0.3307)$ \\
\hline$\Delta \mathrm{q}$ & -2.1763 & 1.3670 & $(-4.8392,0.5149)$ & $\Delta \mathrm{q}$ & 4.6541 & 2.9327 & $(-1.0709,10.441)$ \\
\hline$\Delta \mathrm{b}$ & 0.0044 & 0.0979 & $(-0.1885,0.1986)$ & $\Delta \mathrm{b}$ & 1.5060 & 0.7528 & $(0.0288,2.9841)$ \\
\hline$\Delta \mathrm{r}$ & -0.0869 & 0.1265 & $(-0.3326,0.1645)$ & $\Delta \mathrm{r}$ & -0.0968 & 0.1007 & $(-0.2940,0.1017)$ \\
\hline SP500 & 0.7510 & 0.0956 & $(0.5617,0.9386)$ & SP500 & 1.1706 & 0.1345 & $(0.9016,1.4339)$ \\
\hline \multicolumn{4}{|c|}{$\underline{\text { Microsoft }}$} & \multicolumn{4}{|c|}{ Oracle Corporation } \\
\hline & Mean & Std & $95 \% \mathrm{CI}$ & & Mean & Std & $95 \%$ CI \\
\hline $\mathrm{x}$ & -0.2348 & 0.3546 & $(-0.9304,0.4510)$ & $\mathrm{x}$ & 0.3819 & 0.4432 & $(-0.5117,1.2434)$ \\
\hline$\Delta q$ & 1.1018 & 2.2772 & $(-3.3573,5.5670)$ & $\Delta \mathrm{q}$ & 1.4581 & 2.6409 & $(-3.5877,6.6771)$ \\
\hline$\Delta \mathrm{b}$ & 0.3644 & 0.2323 & $(-0.0993,0.8194)$ & $\Delta \mathrm{b}$ & -0.1382 & 0.1720 & $(-0.4681,0.2028)$ \\
\hline$\Delta \mathrm{r}$ & 0.1579 & 0.1890 & $(-0.2152,0.5212)$ & $\Delta \mathrm{r}$ & -0.0787 & 0.1525 & $(-0.3802,0.2237)$ \\
\hline SP500 & 0.9204 & 0.1158 & $(0.6889,1.1447)$ & SP500 & 1.1977 & 0.1236 & $(0.9521,1.4385)$ \\
\hline \multicolumn{8}{|c|}{ Qualcomm Inc. } \\
\hline & Mean & Std & $95 \%$ CI & & & & \\
\hline $\mathrm{x}$ & -1.9100 & 1.1160 & $(-4.1181,0.2935)$ & & & & \\
\hline$\Delta \mathrm{q}$ & -0.1622 & 6.3623 & $(-12.703,12.321)$ & & & & \\
\hline$\Delta \mathrm{b}$ & 1.8329 & 0.5274 & $(0.8054,2.8707)$ & & & & \\
\hline$\Delta \mathrm{r}$ & -0.4439 & 0.1869 & $(-0.8079,-0.0794)$ & & & & \\
\hline SP500 & 1.0112 & 0.1721 & $(0.6695,1.3405)$ & & & & \\
\hline
\end{tabular}


Table 3 Estimated Coefficients of Observed Factors for the IT Industry from the SUR Model

\begin{tabular}{|c|c|c|c|c|c|c|c|c|}
\hline \multicolumn{3}{|c|}{ Apple Inc. } & \multicolumn{3}{|c|}{ Accenture Plc. } & \multicolumn{3}{|c|}{$\underline{\text { Cisco System }}$} \\
\hline & Estimate & Std & \multicolumn{2}{|c|}{ Estimate } & Std & \multicolumn{2}{|r|}{ Estimate } & Std \\
\hline $\mathrm{x}$ & -1.7051 & 1.9512 & $\mathrm{x}$ & 0.5332 & $0.0840 * *$ & $\mathrm{x}$ & -0.2343 & 0.1501 \\
\hline$\Delta \mathrm{q}$ & -4.3511 & 37.5507 & $\Delta \mathrm{q}$ & 0.1886 & 9.4579 & $\Delta \mathrm{q}$ & 11.3222 & 38.7183 \\
\hline$\Delta \mathrm{b}$ & 1.9415 & $0.5924^{* *}$ & $\Delta \mathrm{b}$ & -0.0312 & $0.0071 * *$ & $\Delta \mathrm{b}$ & -0.1029 & $0.0215^{* *}$ \\
\hline$\Delta \mathrm{r}$ & 0.1013 & 0.0731 & $\Delta \mathrm{r}$ & -0.0782 & 0.0729 & $\Delta \mathrm{r}$ & -0.1836 & $0.0174 * *$ \\
\hline SP500 & 1.2361 & $0.0498 * *$ & SP500 & 0.9312 & $0.0131 * *$ & SP500 & 1.3407 & $0.0178 * *$ \\
\hline \multicolumn{3}{|c|}{$\underline{\text { Dell }}$} & \multicolumn{3}{|c|}{$\underline{\text { EMC corporation }}$} & \multicolumn{3}{|c|}{ Hewlett Packard } \\
\hline & Estimate & Std & & Estimate & Std & & Estimate & Std \\
\hline $\mathrm{x}$ & -0.9095 & $0.1270 * *$ & $\mathrm{x}$ & 0.3842 & 0.3846 & $\mathrm{x}$ & 0.3121 & $0.0146^{* *}$ \\
\hline$\Delta \mathrm{q}$ & -1.9775 & 13.9225 & $\Delta \mathrm{q}$ & -4.0140 & 31.6886 & $\Delta \mathrm{q}$ & 0.4549 & $0.1590 * *$ \\
\hline$\Delta \mathrm{b}$ & -0.1330 & $0.0124 * *$ & $\Delta \mathrm{b}$ & -0.0690 & 0.1097 & $\Delta \mathrm{b}$ & -0.3817 & $0.0731 * *$ \\
\hline$\Delta \mathrm{r}$ & -0.0998 & 0.1083 & $\Delta \mathrm{r}$ & -0.0590 & $0.0078 * *$ & $\Delta \mathrm{r}$ & -0.0217 & $0.0059 * *$ \\
\hline SP500 & 1.3732 & $0.0248 * *$ & SP500 & 1.3175 & $0.0238^{* *}$ & SP500 & 1.1698 & $0.0168 * *$ \\
\hline \multicolumn{3}{|c|}{$\underline{\mathrm{IBM}}$} & \multicolumn{3}{|c|}{ Intel Corporation } & \multicolumn{3}{|c|}{$\underline{\text { Microsoft }}$} \\
\hline & Estimate & Std & & Estimate & Std & & Estimate & Std \\
\hline $\mathrm{x}$ & 0.3421 & $0.0454^{* *}$ & $\mathrm{x}$ & -0.5096 & $0.1018 * *$ & $\mathrm{x}$ & -0.2681 & $0.0808 * *$ \\
\hline$\Delta \mathrm{q}$ & -1.9477 & 1.7203 & $\Delta \mathrm{q}$ & 4.9098 & 7.2429 & $\Delta \mathrm{q}$ & 1.4689 & 4.4320 \\
\hline$\Delta \mathrm{b}$ & 0.0318 & $0.0100 * *$ & $\Delta \mathrm{b}$ & 1.6362 & $0.4953 * *$ & $\Delta \mathrm{b}$ & 0.4137 & $0.0428 * *$ \\
\hline$\Delta \mathrm{r}$ & -0.0836 & $0.0151^{* *}$ & $\Delta \mathrm{r}$ & -0.0979 & $0.0093 * *$ & $\Delta \mathrm{r}$ & 0.1699 & $0.0363 * *$ \\
\hline SP500 & 0.7433 & $0.0091^{* *}$ & SP500 & 1.1644 & $0.0177 * *$ & SP500 & 0.9195 & $0.0132 * *$ \\
\hline \multicolumn{3}{|c|}{ Oracle Corporation } & \multicolumn{3}{|c|}{ Qualcomm Inc. } & & & \\
\hline & Estimate & Std & & Estimate & Std & & & \\
\hline $\mathrm{x}$ & 0.3610 & $0.1575 *$ & $\mathrm{x}$ & -1.9016 & $0.8116^{*}$ & & & \\
\hline$\Delta \mathrm{q}$ & 1.9328 & 6.3625 & $\Delta \mathrm{q}$ & -1.4696 & 34.7751 & & & \\
\hline$\Delta \mathrm{b}$ & -0.1515 & $0.0298 * *$ & $\Delta \mathrm{b}$ & 2.0253 & $0.4528 * *$ & & & \\
\hline$\Delta \mathrm{r}$ & -0.0793 & $0.0203 * *$ & $\Delta \mathrm{r}$ & -0.4426 & $0.0349 * *$ & & & \\
\hline SP500 & 1.1964 & $0.0150 * *$ & SP500 & 1.0009 & $0.0339 * *$ & & & \\
\hline
\end{tabular}

Note: ** indicates $1 \%$ significant, $*$ indicates $5 \%$ significant. 
Table 4 Explaining Power of Observed Factors vs. Unobserved Factors for IT Companies

\begin{tabular}{lcc}
\hline Company & $\operatorname{Var}\left(\mathbf{z}_{i t}^{\prime} \hat{\boldsymbol{\theta}}_{i}\right) / \operatorname{Var}\left(y_{i t}\right)$ & $\operatorname{Var}\left(\hat{\boldsymbol{\lambda}}_{i} \hat{\boldsymbol{f}}_{t}\right) / \operatorname{Var}\left(y_{i t}\right)$ \\
\hline Apple Inc. & 0.2689 & 0.0514 \\
Accenture Plc. & 0.3733 & 0.0208 \\
Cisco System & 0.4788 & 0.0844 \\
Dell & 0.4086 & 0.0808 \\
EMC Corporation & 0.3877 & 0.1570 \\
Hewlett Packard & 0.4350 & 0.0337 \\
International Business Machines & 0.3645 & 0.0532 \\
Intel Corporation & 0.4345 & 0.0921 \\
Microsoft & 0.3861 & 0.0157 \\
Oracle Corporation & 0.4610 & 0.0846 \\
Qualcomm Inc. & 0.2984 & 0.0273 \\
Overall & $\mathbf{0 . 3 8 5 0}$ & $\mathbf{0 . 0 6 5 5}$ \\
\hline
\end{tabular}

Table 5 MSE's from Bayesian Common Factor Model with Different Specifications on a Number of Industry-Wide Common Factors

\begin{tabular}{lccccc}
\hline Model & IT & Industrial & Energy & Finance & Health Care \\
\hline One factor & 0.0034 & 0.0030 & 0.0045 & 0.0053 & 0.0031 \\
Two factors & 0.0034 & 0.0026 & 0.0038 & 0.0045 & 0.0029 \\
Three factors & 0.0034 & 0.0025 & 0.0040 & 0.0044 & 0.0028 \\
\hline
\end{tabular}


Figure 1 MCMC Sample Paths and ACFs of Coefficients Estimated from Model CF for Apple Inc.
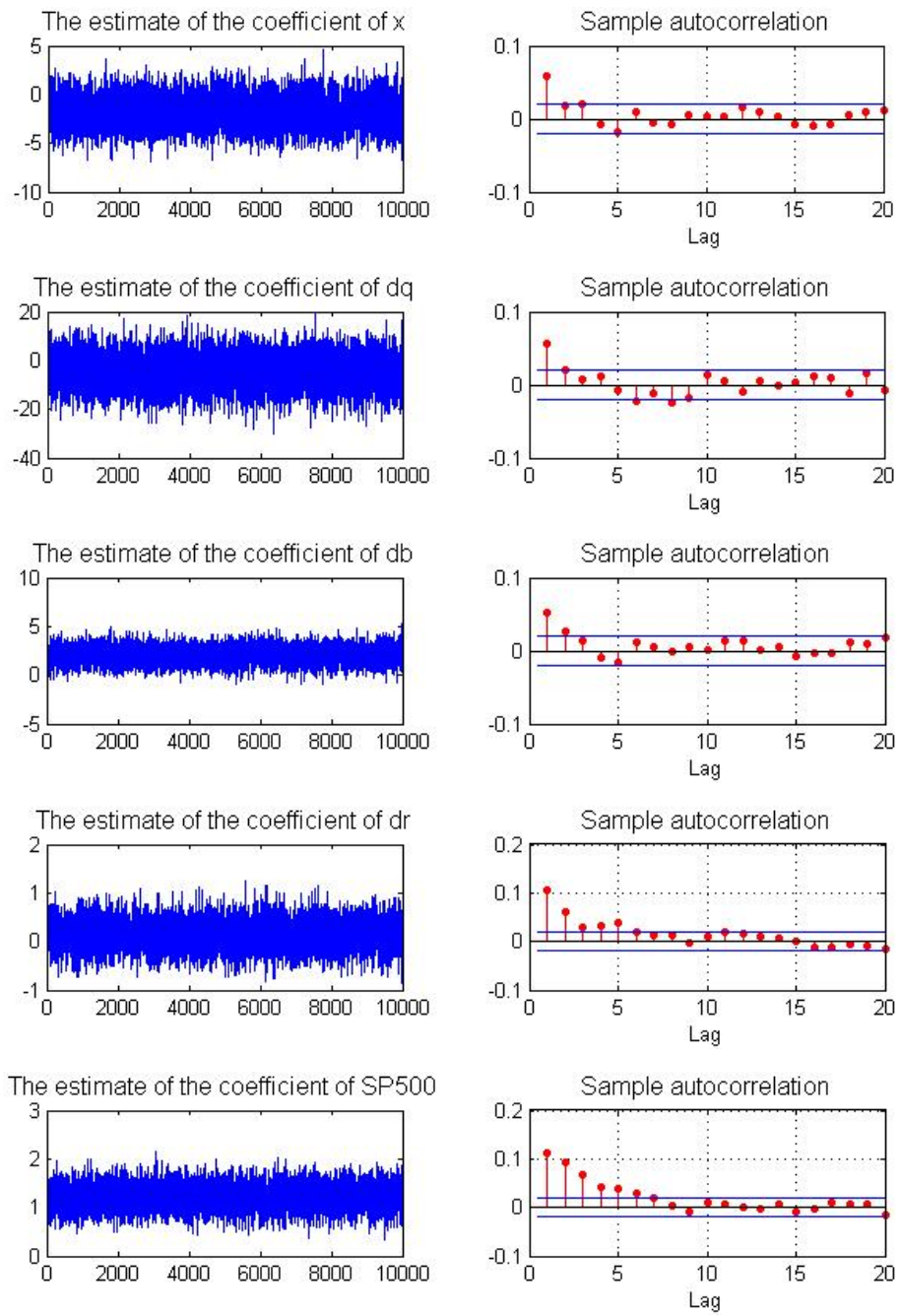


\section{References}

Bai, J (2009). “Panel Data Models with Interactive Fixed Effects.” Econometrica, 77, 1229-1279.

Bettman, J., S. Sault and E. Schultz (2009). "Fundamental and Technical Analysis: Substitutes or Complements?” Accounting and Finance, 49, 21-36.

Breusch, T. S., and A. R. Pagan (1980), "The Lagrange Multiplier Test and its Application to Model Specifications in Econometrics”. Review of Economic Studies, 47, 239-53.

Chan, J. C. C., R. Leon-Gonzalez and R. W. Strachan (2013). "Invariant Inference and Efficient Computation in the Static Factor Model." CAMA Working Papers.

Chen, P. and G. Zhang (2007). "How Do Accounting Variables Explain Stock Price Movements? Theory and Evidence.” Journal of Accounting and Economics, 42, 219-244.

Clement, M., J. Hales and Y. Xue (2011). "Understanding Analysts' Use of Stock Returns and Other Analysts' Revisions when Forecasting Earnings.” Journal of Accounting and Economics, 51, 279-299

Geweke, J. and G. F. Zhou (1996). "Measuring the Pricing Error of the Arbitrage Pricing Theory.” Review of Financial Studies, 9, 557-587.

Hao, S., Q. Jin and G. Zhang (2011). "Relative Firm Profitability and Stock Return Sensitivity to IndustryLevel News.” The Accounting Review, 86, 1321-1347.

Harford, J. (2005). “What Drives Merge Waves?” Journal of Financial Economics, 77, 529-560.

Hong, K. and E. Wu (2014), “Can Momentum Factors be used to Enhance Accounting Information based Fundamental Analysis in Explaining Stock Price Movements?” Quantitative Finance Research Centre Working Paper Series, 342.

Lessard, D.R. (1974). "World, National and Industry Factors in Equity Returns.” Journal of Finance, 29, 379-391.

Pesaran, M. H. (2006). "Estimation and Inference in Large Heterogeneous Panels with a Multifactor Error Structure.” Econometrica, 74, 967-1012.

Sarafidis, V. and T. Wansbeek (2012). “Cross-Sectional Dependence in Panel Data Analysis.” Econometric Reviews, 31, 483-531.

Zellner, A. (1962). “An Efficient Method of Estimating Seemingly Unrelated Regression Equations and Tests of Aggregation Bias.” Journal of the American Statistical Association, 57, 500-509

Zhang, G. (2000). “Accounting Information, Capital Investment Decisions, and Equity Valuation: Theory and Empirical Implications.” Journal of Accounting Research, 38, 271-295 


\section{Appendix}

Table A1 Estimated Heterogeneous Coefficients of Observed Factors for Selected Companies by SUR Model, Bayesian estimator and CCE Estimator

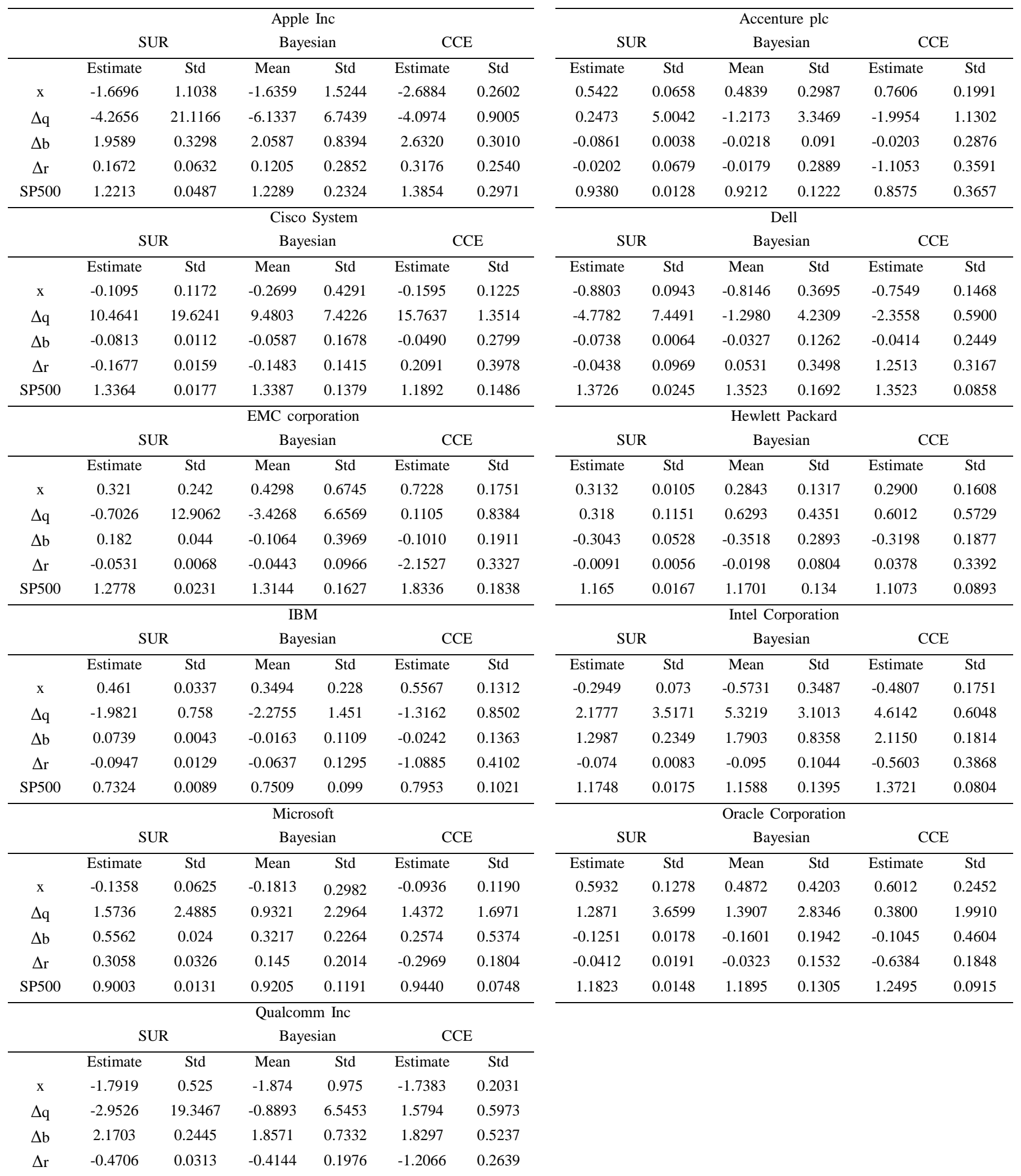

Note: The three models are estimated for pooled data. Results reported in this table are for selected IT companies. 
Table A2 Estimated Homogeneous Coefficients of Observed Factors for all companies by CCEP Estimator, CCEMG estimator and IPC estimator for Factor Structure Model

\begin{tabular}{lccccccccccc}
\hline & \multicolumn{2}{c}{ CCEP } & \multicolumn{2}{c}{ CCEMG } & \multicolumn{2}{c}{ IPC (one factor) } & \multicolumn{2}{c}{ IPC (two factors) } & \multicolumn{2}{c}{ IPC (three factors) } \\
& Estimate & Std & Estimate & Std & Estimate & Std & Estimate & Std & \multicolumn{2}{c}{ Estimate } & Std \\
\hline $\mathrm{x}$ & 0.0640 & 0.3174 & -0.0755 & 0.2795 & 0.0108 & 0.0162 & -0.0264 & 0.2325 & 0.0281 & 0.0344 \\
$\Delta \mathrm{q}$ & 0.1024 & 1.3262 & -1.3843 & 1.8118 & -0.0036 & 0.0129 & -0.0158 & 0.5376 & 0.0557 & 0.0068 \\
$\Delta \mathrm{b}$ & 0.0360 & 0.2920 & 0.0705 & 0.3086 & 0.0007 & 0.0001 & 0.0008 & 0.2476 & 0.0013 & 0.0001 \\
$\Delta \mathrm{r}$ & -0.0822 & 0.2619 & -0.2073 & 0.2813 & -0.0246 & 0.0020 & -0.0234 & 0.0165 & -0.0129 & 0.0015 \\
SP500 & 1.1001 & 0.0864 & 1.1924 & 0.0766 & 1.0465 & 0.0009 & 1.0398 & 0.0228 & 0.9254 & 0.0026 \\
\hline
\end{tabular}

